\title{
Reoperation for Bleeding After Thyroid and Parathyroid Surgery: Incidence, Risk Factors, Prevention, and Management
}

\author{
O. Edafe $^{1,3} \cdot$ E. Cochrane ${ }^{2} \cdot$ S. P. Balasubramanian ${ }^{2,3}$
}

Published online: 10 December 2019

(C) The Author(s) 2019

\begin{abstract}
Introduction Bleeding after thyroid and/or parathyroid surgery is a life-threatening emergency. The aim of this study was to determine the rates of reoperation following bleeding, identify risk factors, assess management strategies and outcomes, and develop protocols to reduce risk and improve management of bleeding.

Methods A retrospective cohort study of all consecutive patients who underwent thyroid and/or parathyroid surgery over a 7-year period was conducted. A nested case-control design was used to evaluate specific factors and their association with reoperation for bleeding.

Results Of 1913 patients, 25 (1.3\%) underwent reoperation for bleeding. Of the 25 patients who bled, 6 (24\%) required reoperation before leaving theatre; $17(68 \%)$ had bleeding within $6 \mathrm{~h}, 1(4 \%)$ between 6 and $24 \mathrm{~h}$, and 1 (4\%) after $24 \mathrm{~h}$. Reoperation for bleeding was not associated with age, gender, or surgeon. Patients who had total thyroidectomy were more likely to have reoperation for bleeding compared to hemithyroidectomy $(p=0.045)$ or parathyroidectomy $(p=0.001)$. The following factors were not associated with bleeding: neck dissection, re-do surgery, drain use, blood-thinning medication or clotting disorders, and BMI. Patients who had reoperation for bleeding had longer hospital stay $(p=0.001)$, but similar rates of RLN palsy, wound infection, and hypoparathyroidism.

Conclusion A higher risk profile for significant post-operative bleeding cannot be determined in patients undergoing thyroid surgery. Based on this experience, we developed protocols to reduce the risk of bleeding (the ITSRED Fred protocol) and for the early detection and management of bleed (the SCOOP protocol) following thyroid and/or parathyroid surgery.
\end{abstract}

O. Edafe and E. Cochrane: Co-first author.

O. Edafe

ovieedafe@hotmail.co.uk

1 Department of ENT, Sheffield Teaching Hospitals NHS Foundation Trust, Sheffield, UK

2 Endocrine Surgery Unit, Department of General Surgery, Sheffield Teaching Hospitals NHS Foundation Trust, Sheffield, UK

3 Department of Oncology and Metabolism, University of Sheffield, Beech Hill Road, Sheffield S10 2RX, UK

\section{Introduction}

Bleeding after thyroid or parathyroid surgery is a lifethreatening emergency as it has the potential to compromise the airway [1]. Reoperation for bleeding can be considered an objective way of quantifying rates of significant bleeding and has been reported to occur in around 0.07 to $4.2 \%$ of such neck operations [1-20].

Identification of risk factors for bleeding may help to identify patients requiring closer monitoring and guide development of preventative measures. A Medline search showed a number of articles directed at incidence and risk 
factors [2-6]; but there are little data on preventative and management strategies.

Post-thyroidectomy haematoma can potentially lead to significant morbidity including emergency tracheostomy, cardiac arrest, stroke, and transient ischaemic attack [3, 4]. Although rare, deaths attributable to post-thyroidectomy haematoma have also been reported [3-5, 7].

The aim of this study was to determine the incidence of post-operative bleeding in patients undergoing thyroid or parathyroid surgery in one tertiary centre, identify risk factors for bleeding, study bleeding patterns, and evaluate management strategies and outcomes. In addition, we sought to devise a preventative algorithm for neck haematoma, and a protocol for early detection and management of neck haematoma.

\section{Methods}

\section{Study design}

This was primarily an observational retrospective cohort study. In addition, a nested case-control analysis was also performed to study a few additional risk factors in more detail. In this analysis, 197 patients were selected randomly from the non-bleed group, and this was compared to the 25 patients in the bleed group.

\section{Setting}

The study was undertaken at Sheffield Teaching Hospitals' endocrine surgical unit, a tertiary referral site. All patients who underwent thyroid and/or parathyroid surgery between January 2010 and March 2017 were included. All operations were performed or supervised by one of two endocrine surgical consultants in the unit; however, four different consultants were involved in the study period. Data were collected using a standardised data collection tool.

\section{Participants}

All consecutive patients who underwent thyroid and/or parathyroid surgery were included. In addition, patients who underwent lymph node dissection without thyroidectomy/parathyroidectomy were also included. Patients undergoing excision of thymic cysts or biopsy/excision of lymph nodes or lipomata in the neck were excluded. Eligible patients were identified using theatre electronic database.

\section{Variables}

The primary outcome was the rate of reoperation for postoperative bleeding. This was defined by the need for evacuation of haematoma either as part of the same operation (after skin closure) or a return to operating theatre. The following secondary outcomes were evaluated: transient and permanent recurrent laryngeal nerve (RLN) palsy, transient and long-term hypoparathyroidism, and cultureproven wound infection. RLN palsy was confirmed on post-operative laryngoscopy. This was considered transient if the patients' voice returned completely to normal or the patient had a normal repeat vocal cord check at subsequent follow-up. Transient hypoparathyroidism was defined as post-operative day one corrected calcium less than $2.10 \mathrm{mmol} / \mathrm{L}$. Long-term hypoparathyroidism was defined as the need for calcium and/or vitamin D supplements to maintain normocalcaemia at 6 months or more after surgery.

In the entire cohort, factors such as age, gender, and primary organ of surgery were compared between those who underwent reoperation for bleeding and those who did not. The following factors were also evaluated as part of a nested case-control study, as described previously: primary surgery, neck dissection, re-do surgery, drain use, bloodthinning medication use or clotting disorder, RLN palsy, wound infection, hypoparathyroidism, BMI, and length of stay.

\section{Data collection and analysis}

Data were collected from electronic patient databases (theatre databases, electronic reporting, electronic case notes and letters) into a Microsoft Excel $^{\mathrm{TM}}$ spreadsheet. No formal sample size calculations were done as all consecutive patients within the defined inclusion period were included. The data were anonymised and transferred to SPSS for analyses. Categorical data were reported as frequencies. Parametric data were reported as mean and standard deviation, and nonparametric data as median and interquartile range. The association between variables and post-operative haematoma was analysed with Chi-squared test (or Fishers' exact) for categorical variables, and independent sample $t$ test for (or Mann-Whitney $U$ test) for continuous variables. A $p$ value of $<0.05$ was considered to be statistically significant.

Approval from the study was obtained from the clinical effectiveness in Sheffield Teaching Hospitals (Ref No. 9635). As this was an observational study, a formal ethics application was not deemed to be necessary and individual patient consent was not obtained. All data were initially stored in password-protected NHS computers, and patient 
identifiable data were removed prior to analyses and reporting.

\section{Results}

A total number of 1913 procedures were performed. The operations were as follows: $1253(65.5 \%)$ thyroid surgery, $613(32 \%)$ parathyroid surgery, and $47(2.5 \%)$ concomitant thyroid and parathyroid surgery. There were $1454(76 \%)$ female and $459(24 \%)$ male patients, with a median age of 53 (i.q.r. 27) years.

Of the 1913 procedures, 25 (1.3\%) patients had reoperation for bleeding. The rates were $1.8 \%$ (23/1253) in thyroid surgery, $0.3 \%(2 / 613)$ in parathyroid surgery, and $0 \%(0 / 47)$ in patients who had both thyroid and parathyroid surgery. Patients who underwent thyroidectomy were more likely to have reoperation for bleeding compared to those that underwent parathyroidectomy $(p=0.008)$. The consultant involved, age of the patient at surgery, and patient gender were not associated with reoperation for bleeding (Table 1).

Further analyses were performed as part of the nested case-control study. We compared the 25 patients who had reoperation for bleeding to 197 randomly selected patients with no bleeding (see Table 2). Patients who had total thyroidectomy were more likely to have reoperation for bleeding compared to hemithyroidectomy $(p=0.045)$ or parathyroidectomy ( $p=0.001)$. Patients who had reoperation were significantly more likely to have a longer hospital stay $(p=0.001)$.

Table 1 Association between clinical characteristics and reoperation for bleeding for whole cohort

\begin{tabular}{lccc}
\hline Characteristics & \multicolumn{2}{c}{ Reoperation for bleeding } & \multirow{2}{*}{$p$ value } \\
\cline { 2 - 3 } & Yes (25) & No (1888) & \\
\hline Age, median (i.q.r.) & $52(35)$ & $53(26)$ & 0.986 \\
Gender & & & \\
Female & $18(1.2 \%)$ & $1436(98.8 \%)$ & 0.639 \\
Male & $7(1.5 \%)$ & $452(98.5 \%)$ & \\
Primary organ of surgery & & & \\
Thyroid & $23(1.8 \%)$ & $1230(98.2 \%)$ & 0.017 \\
Parathyroid & $2(0.3 \%)$ & $611(99.7 \%)$ & \\
Both thyroid and parathyroid & $0(0 \%)$ & $47(100 \%)$ & \\
Consultants & & & \\
A & $13(1.5 \%)$ & $840(98.5 \%)$ & 0.255 \\
B & $8(0.9 \%)$ & $874(99.1 \%)$ & \\
C & $4(2.6 \%)$ & $147(97.4 \%)$ & \\
D & $0(0 \%)$ & $27(100 \%)$ & \\
\hline
\end{tabular}

i.q.r., interquartile range
Table 3 shows the management strategies in the 25 patients who had reoperation for bleeding. In 23 patients $(92.0 \%)$, bleeding occurred either in the operating theatre after skin closure or less than $6 \mathrm{~h}$ after surgery. The management included removal of neck sutures on the ward (or post-anaesthesia care unit) prior to definitive exploration in $6 / 19(31.6 \%)$ and steroid administration in 12/19 (63.2\%). The source of bleeding was clearly identifiable in $16 / 25$ $(64.0 \%)$ of patients. A neck drain was used after exploration for bleeding in $18 / 25(72.0 \%)$ patients, and $8 / 25$ $(32.0 \%)$ patients were monitored in high-dependency unit following surgery. One patient required blood transfusion. There was no post-operative mortality or need for tracheostomy in the cohort of patients who underwent reoperative surgery for bleeding.

\section{Discussion}

\section{Incidence and risk factors for bleeding after thyroid and/or parathyroid surgery}

This study examined the incidence and risk factors for significant bleeding (defined as need for reoperation) following thyroid and parathyroid surgery. The management of patients with significant bleeding and their clinical outcomes have also been described. We also included patients who had bleeding in theatre after skin closure. The reoperation rate for bleeding in our unit was $1.3 \%$. This falls within the range reported in the literature $(0.07$ to $4.2 \%$ ) [1-20]. Generally, lower rates of reoperation for bleeding have been reported in patients undergoing parathyroid surgery alone (0 to $1.1 \%)$ [2, 11, 16, 18, 19] or when rates in parathyroid surgery and thyroid surgery are pooled $(0.4$ to $0.8 \%)$ [2, 11, 16, 19].

We found that total thyroidectomy significantly increases the risk of reoperation for bleeding compared to hemithyroidectomy and parathyroidectomy. This is in keeping with other reports [4, 21]. Furthermore, other studies have identified older age [4, 5, 21], male gender [4, 5, 21], antithrombotic medication [21], bleeding disorders [5], neck dissection [21], and re-do operation [4, 21] as risk factors for bleeding. These variables were not associated with bleeding in our cohort.

The use of haemostatic agents in our cohort was variable and was not systematically documented in the operative notes, so we did no evaluate this. Other studies have found no effect in the rates of bleeding with the use haemostatic gel [22], oxidised cellulose patch [23], and fibrin sealant (TachoSil) [24]. 
Table 2 Association between clinical characteristics and reoperation for bleeding in the nested case-control study

\begin{tabular}{|c|c|c|c|}
\hline \multirow[t]{2}{*}{ Characteristics } & \multicolumn{2}{|c|}{ Reoperation for bleeding } & \multirow[t]{2}{*}{$p$ value } \\
\hline & Yes $(25)$ & No (197) & \\
\hline \multicolumn{4}{|l|}{ Index procedure } \\
\hline TT & $16(64.0 \%)$ & $60(30.5 \%)$ & \multirow[t]{4}{*}{0.006} \\
\hline HT & $7(28.0 \%)$ & $68(34.5 \%)$ & \\
\hline Parathyroidectomy & $2(8.0 \%)$ & $66(33.5 \%)$ & \\
\hline Others & $3(1.4 \%)$ & $3(1.5 \%)$ & \\
\hline \multicolumn{4}{|l|}{ Neck dissection } \\
\hline Yes & $2(8.0 \%)$ & $15(7.6 \%)$ & \multirow[t]{2}{*}{1.000} \\
\hline No & $23(92.0 \%)$ & $182(92.4 \%)$ & \\
\hline \multicolumn{4}{|l|}{ Re-do surgery } \\
\hline Yes & $1(4.0 \%)$ & $14(7.1 \%)$ & \multirow[t]{2}{*}{1.000} \\
\hline No & $24(96.0 \%)$ & $183(92.9 \%)$ & \\
\hline \multicolumn{4}{|l|}{ Drain use ${ }^{c}$} \\
\hline Yes & $3(12.5 \%)$ & $16(8.1 \%)$ & \multirow[t]{2}{*}{0.442} \\
\hline No & $21(87.5 \%)$ & $181(91.9 \%)$ & \\
\hline \multicolumn{4}{|l|}{ Any blood-thinning drugs or clotting disorder ${ }^{c}$} \\
\hline Yes & $2(8.3 \%)$ & $24(12.2 \%)$ & \multirow[t]{2}{*}{0.747} \\
\hline No & $22(91.7 \%)$ & $173(87.8 \%)$ & \\
\hline \multicolumn{4}{|l|}{ RLN palsy $^{\mathrm{c}}$} \\
\hline None & $20(95.2 \%)$ & $177(94.7 \%)$ & \multirow[t]{3}{*}{0.699} \\
\hline Transient & $0(0 \%)$ & $4(2.1 \%)$ & \\
\hline Permanent & $1(4.8 \%)$ & $6(3.2 \%)$ & \\
\hline \multicolumn{4}{|l|}{ Wound infection } \\
\hline Yes & $0(0 \%)$ & $2(1.0 \%)$ & \multirow[t]{2}{*}{1.000} \\
\hline No & $25(100 \%)$ & $195(99.0 \%)$ & \\
\hline \multicolumn{4}{|l|}{ Hypoparathyroidism $^{\mathrm{c}}$} \\
\hline None & $16(84.2 \%)$ & $168(92.3 \%)$ & \multirow[t]{3}{*}{0.184} \\
\hline Transient & $1(5.3 \%)$ & $9(4.9 \%)$ & \\
\hline Long term & $2(10.5 \%)$ & $5(2.7 \%)$ & \\
\hline \multicolumn{4}{|l|}{ Significant complication } \\
\hline None & $21(84.0 \%)$ & $174(88.3 \%)$ & \multirow[t]{3}{*}{0.422} \\
\hline Transient $^{\mathrm{a}}$ & $1(4.0 \%)$ & $12(6.1 \%)$ & \\
\hline Long term ${ }^{\mathrm{b}}$ & $3(12.0 \%)$ & $11(5.6 \%)$ & \\
\hline BMI, median (i.q.r.) & $27(24-29)$ & $27(23-31)$ & 0.848 \\
\hline Length of stay after surgery, days, median (i.q.r.) & $3(2-3)$ & $1(1-2)$ & 0.001 \\
\hline
\end{tabular}

i.q.r., interquartile range; BMI, body mass index

${ }^{a}$ Hypoparathyroidism, RLN palsy, wound infection

${ }^{\mathrm{b}} \mathrm{RLN}$ palsy, hypoparathyroidism

${ }^{\mathrm{c}}$ Missing data excluded from analysis; TT, total thyroidectomy; HT, hemithyroidectomy; RLN, recurrent laryngeal nerve

\section{Prevention of bleeding after thyroid and/ or parathyroid surgery}

In our study, six patients had bleeding whilst in theatre after wound closure. We found clear sources of bleeding in five of these patients (arterial/venous/thyroid bed). This can be attributed to many factors including inadequate haemostasis; slippage of clips/knots as a result of increased venous pressure from coughing/retching at extubation or elevated blood pressure at extubation. This experience has led us to develop an intraoperative protocol to prevent bleeding (ITSRED Fred) (Table 4). The protocol includes practical steps to identify bleeding points prior to wound closure: irrigation with water to remove blood clots and 
Table 3 Management of 25 patients who had reoperation for bleeding after thyroid and/or parathyroid surgery

\begin{tabular}{|c|c|}
\hline Management characteristics & $n(\%)$ \\
\hline \multicolumn{2}{|l|}{ Time bleeding identified } \\
\hline In theatre & $6 / 25(24)$ \\
\hline After leaving theatre $(\leq 6 \mathrm{~h})$ & $17 / 25(68)$ \\
\hline 6-24 h post-operative & $1 / 25(4)$ \\
\hline$>24 \mathrm{~h}$ & $1 / 25(4)$ \\
\hline \multicolumn{2}{|l|}{ Neck suture removed prior to theatre ${ }^{a}$} \\
\hline Yes & $6 / 19(31.6)$ \\
\hline No & $11 / 19(57.9)$ \\
\hline Unknown & $2 / 19(10.5)$ \\
\hline \multicolumn{2}{|l|}{ Steroids given ${ }^{\mathrm{b}}$} \\
\hline Yes & $12 / 19(63.2)$ \\
\hline No & $6 / 19(31.6)$ \\
\hline NK & $1 / 19(5.3)$ \\
\hline \multicolumn{2}{|l|}{ Bleeding points ${ }^{\mathrm{c}}$} \\
\hline Pyramidal lobe vessel & $1(4)$ \\
\hline Strap muscles/sternocleidomastoid & $3(12)$ \\
\hline Thyroid bed & $2(8)$ \\
\hline Inferior thyroid artery/branch & $3(12)$ \\
\hline Cricothyroid junction & $2(8)$ \\
\hline Anterior jugular vein & $1(4)$ \\
\hline Superior pole vessel & $3(12)$ \\
\hline Superior thyroid vein & $1(4)$ \\
\hline Generalised ooze & $1(4)$ \\
\hline Ligament of Berry & $1(4)$ \\
\hline No specific bleeding point & $9(36)$ \\
\hline \multicolumn{2}{|l|}{ Imaging done } \\
\hline Ultrasound neck ${ }^{\mathrm{b}}$ & $1 / 25(4)$ \\
\hline No imaging & $24 / 25(96)$ \\
\hline \multicolumn{2}{|l|}{ Drain use following washout } \\
\hline Yes & $18 / 25(72.0)$ \\
\hline No & $7 / 25(28.0)$ \\
\hline \multicolumn{2}{|l|}{ Blood transfusion } \\
\hline Yes & $1 / 25(4.0)$ \\
\hline No & $24 / 25(96.0)$ \\
\hline \multicolumn{2}{|l|}{ HDU care after surgery } \\
\hline Yes & $8 / 25(32.0)$ \\
\hline No & $17 / 25(68.0)$ \\
\hline
\end{tabular}

${ }^{a}$ Only includes patients who bled after leaving theatre

${ }^{\mathrm{b}}$ One patient had ultrasound after presenting 4 days post-operative

${ }^{\mathrm{c}}$ Two cases with multiple bleeding points

identify other bleeding points, pause to check for bleeding, elevation of systolic BP to over $100 \mathrm{~mm} \mathrm{Hg}$, relief of neck extension, elevation of venous pressure (either by a Valsalva manoeuvre, by 'head-down' position or both)
[25-27], use of adjuncts (such as regenerated cellulose) $[25,26]$, and a final assessment of bleeding under the flaps.

\section{Management of bleeding after thyroid and/ or parathyroid surgery}

Furthermore, during the study period, there was no readily available formal protocol for use by junior staff for the management for neck haematoma. Our experience of managing the 25 patients who had bleeding has led to the development of a protocol to aid early detection and management of neck swelling post-thyroid and parathyroid surgery (Fig. 1). This involves initiating early oxygen therapy, intravenous hydrocortisone, and immediate involvement of the surgeon, anaesthetist and lead theatre nurse. In cases of significant airway compromise and the absence of trained staff to intubate, removal of sutures and relieving pressure in the recovery room or surgical ward serves as a temporising measure before definitive surgical exploration. Inability to secure the airway via endotracheal intubation may result in tracheostomy or cricothyroidotomy [26].

The occurrence of significant bleeding $6 \mathrm{~h}$ after surgery is cited as a concern in the routine adoption of day case thyroid surgery [28]. Given most bleeds occur within $24 \mathrm{~h}$ after surgery, some have argued for at least $24 \mathrm{~h}$ of observation [13]. Others have argued that day case thyroid surgery is feasible with appropriate patient selection, patient support system, and experienced surgical teams [19]. Rajeev et al. [19] retrospectively evaluated patients who underwent day case thyroid and parathyroid surgery. A total of 163/1326 (12.3\%) thyroid surgery and 275/776 (35.4\%) parathyroid operations were performed as day case. None of the day case patients were re-admitted for bleeding. Based on our experience, parathyroidectomy can be performed as a day case procedure in appropriately selected patients given the low rates of reoperation for bleeding. However, we are unable to stratify risk of reoperation for bleeding in thyroid surgery.

\section{Limitations}

The limitations of this study primarily include its retrospective nature and the biases inherent to these types of study. However, selection bias was minimised by ensuring that all consecutive patients within the study period were included. Any bias in ascertainment of outcomes was minimised by ensuring that these were defined as objectively as possible and the data were collected independently of the operating surgeons. The definition of postoperative haematoma/bleeding may be variable and subjective; which is why it has been defined as 'need for reoperation' in this study. Given the rarity of the primary 
Table 4 ITSRED Fred Protocol for preventing post-surgical neck haematoma after thyroid and/or parathyroid surgery

\begin{tabular}{ll}
\hline & Parameters \\
\hline $\mathrm{I}$ & Irrigation of wound with water-to clear field and look for minor bleeding points [11, 25] \\
$\mathrm{T}$ & Time-wait a few minutes \\
$\mathrm{R}$ & Systolic blood pressure to be $>100 \mathrm{~mm} \mathrm{Hg}$ at time of closure \\
$\mathrm{E}$ & Relieve neck extension-either by deflating bag under shoulders, removing sand bag, or flexing the neck \\
$\mathrm{D}$ & Elevate venous pressure-either by Valsalva manoeuvre [25, 26], head-down position, or both [27] \\
Fred & Do not drain it, deal with it and consider sealants such as fibrillar [25, 26] \\
\hline
\end{tabular}

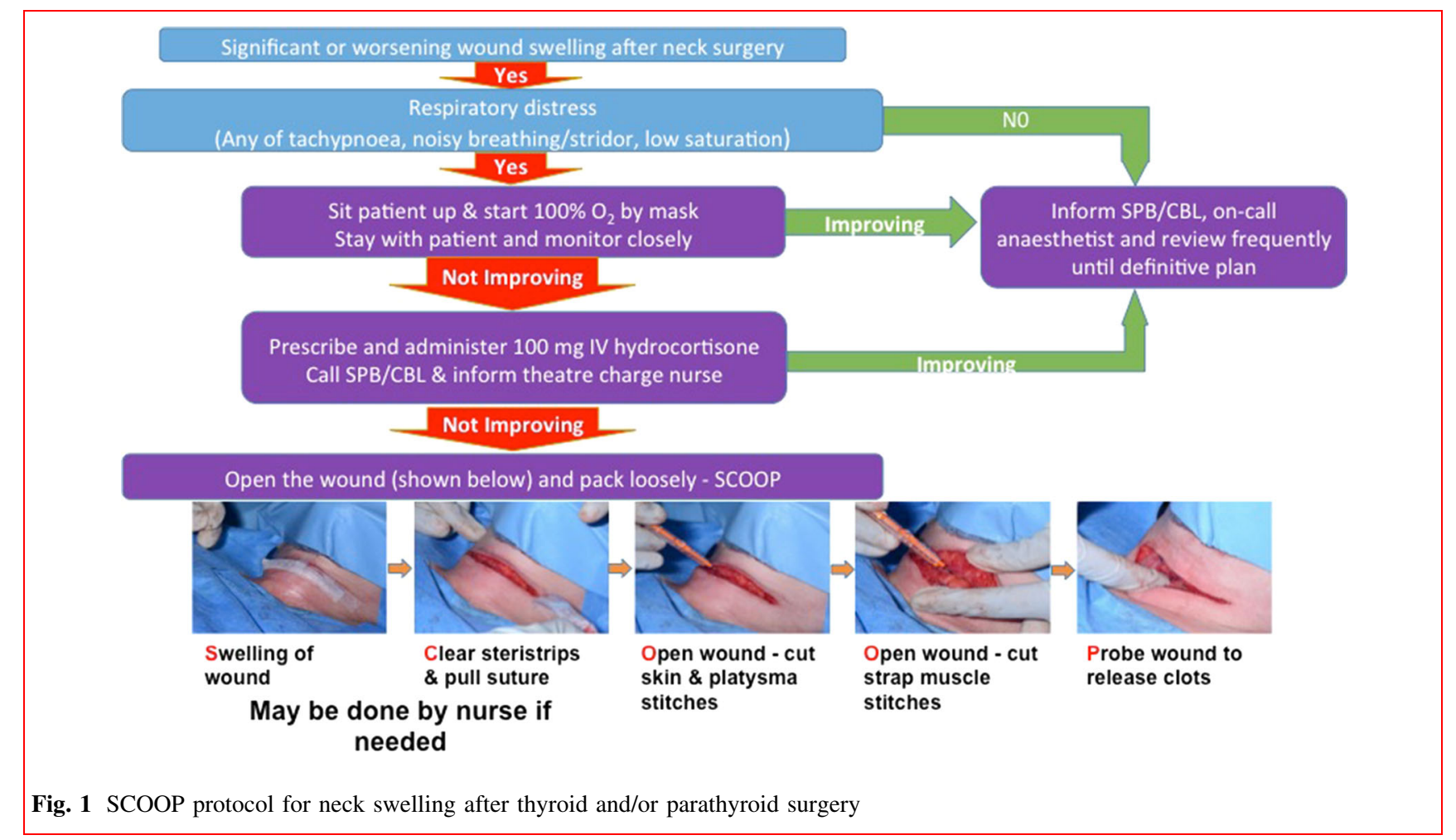

outcome, the analyses of risk factors could be susceptible to type II error. In addition, missing data on RLN palsy and hypoparathyroidism in the 'no bleeding cohort' limited assessment of these variables. Although we have developed protocols for the prevention (Table 4) and management (Fig. 1) of haematoma following neck surgery, these have not been validated and their effectiveness is as yet unclear.

\section{Conclusion}

The rate of neck haematoma following thyroid and parathyroid surgery in our unit is comparable to others. Risk stratification for bleeding in thyroid surgery could not be achieved based on the results. Further work should assess the effectiveness of preventative and management strategies to reduce morbidity of neck haematoma.

\section{Compliance with ethical standards}

Conflict of interest The authors declare that they have no conflict of interest.

Open Access This article is licensed under a Creative Commons Attribution 4.0 International License, which permits use, sharing, adaptation, distribution and reproduction in any medium or format, as long as you give appropriate credit to the original author(s) and the source, provide a link to the Creative Commons licence, and indicate if changes were made. The images or other third party material in this article are included in the article's Creative Commons licence, unless indicated otherwise in a credit line to the material. If material is not included in the article's Creative Commons licence and your intended 
use is not permitted by statutory regulation or exceeds the permitted use, you will need to obtain permission directly from the copyright holder. To view a copy of this licence, visit http://creativecommons. org/licenses/by/4.0/.

\section{References}

1. Rosenbaum MA, Haridas M, McHenry CR (2008) Life-threatening neck hematoma complicating thyroid and parathyroid surgery. Am J Surg 195:339-343

2. Abbas G, Dubner S, Heller KS (2001) Re-operation for bleeding after thyroidectomy and parathyroidectomy. Head Neck 23:544-546

3. Wojtczak B, Aporowicz M, Kaliszewski K et al (2018) Consequences of bleeding after thyroid surgery-analysis of 7805 operations performed in a single center. Arch Med Sci 14:329-335

4. Promberger R, Ott J, Kober F et al (2012) Risk factors for postoperative bleeding after thyroid surgery. $\mathrm{Br} \quad \mathrm{J}$ Surg 99:373-379

5. Weiss A, Lee KC, Brumund KT et al (2014) Risk factors for hematoma after thyroidectomy: results from the nationwide inpatient sample. Surgery 156:399-404

6. Sorensen KR, Klug TE (2015) Routine outpatient thyroid surgery cannot be recommended. Dan Med J 62:A5016

7. Bergamaschi R, Becouarn G, Ronceray J et al (1998) Morbidity of thyroid surgery. Am J Surg 176:71-75

8. Materazzi G, Dionigi G, Berti P et al (2007) One-day thyroid surgery: retrospective analysis of safety and patient satisfaction on a consecutive series of 1571 cases over a three-year period. Eur Surg Res 39:182-188

9. Lang BHH, Yih PCL, Lo CY (2012) A review of risk factors and timing for postoperative hematoma after thyroidectomy: is outpatient thyroidectomy really safe? World J Surg 36:2497-2502

10. Godballe C, Madsen AR, Pedersen HB et al (2009) Post-thyroidectomy hemorrhage: a national study of patients treated at the Danish departments of ENT Head and Neck Surgery. Eur Arch Otorhinolaryngol 266:1945-1952

11. Dixon JL, Snyder SK, Lairmore TC et al (2014) A novel method for the management of post-thyroidectomy or parathyroidectomy hematoma: a single-institution experience after over 4000 central neck operations. World J Surg 38:1262-1267

12. Segel JM, Duke WS, White JR et al (2016) Outpatient thyroid surgery: safety of an optimized protocol in more than 1000 patients. Surgery 159:518-523

13. Farooq M, Nouraei R, Kaddour $\mathrm{H}$ et al (2017) Patterns, timing and consequences of post-thyroidectomy haemorrhage. Ann R Coll Surg Engl 99:60-62

14. Bergenfelz A, Jansson S, Kristoffersson A et al (2008) Complications to thyroid surgery: results as reported in a database from a multicenter audit comprising 3660 patients. Langenbecks Arch Surg 393:667-673

15. Zhang X, Du W, Fang Q (2017) Risk factors for postoperative haemorrhage after total thyroidectomy: clinical results based on 2678 patients. Sci Rep 7:7075

16. Talutis SD, Drake FT, Sachs T et al (2019) Evacuation of postoperative hematomas after thyroid and parathyroid surgery: an analysis of the CESQIP Database. Surgery 165:250-256

17. Perera M, Anabell L, Page D et al (2016) Risk factors for postthyroidectomy haematoma. J Laryngol Otol 130(Suppl 1):S20 S25

18. Tang JA, Salapatas AM, Bonzelaar LB et al (2017) Parathyroidectomy for the treatment of hyperparathyroidism: thirty-day morbidity and mortality. Laryngoscope 128:528-533

19. Rajeev P, Sutaria R, Ezzat T et al (2014) Changing trends in thyroid and parathyroid surgery over the decade: is same-day discharge feasible in the United Kingdom? World J Surg 38:2825-2830

20. Friguglietti CU, Lin CS, Kulcsar MA (2003) Total thyroidectomy for benign thyroid disease. Laryngoscope 113:1820-1826

21. Liu J, Sun W, Dong W et al (2017) Risk factors for post-thyroidectomy haemorrhage: a meta-analysis. Eur $\mathrm{J}$ Endocrinol 176:591-602

22. Khadra H, Bakeer M, Hauch A et al (2018) Hemostatic agent use in thyroid surgery: a meta-analysis. Gland Surg 7:S34-S41

23. Amit M, Binenbaum Y, Cohen JT et al (2013) Effectiveness of an oxidized cellulose patch hemostatic agent in thyroid surgery: a prospective, randomized, controlled study. J Am Coll Surg 217:221-225

24. Erdas E, Medas F, Podda F et al (2015) The use of a biologic topical haemostatic agent (TachoSil((R))) for the prevention of postoperative bleeding in patients on antithrombotic therapy undergoing thyroid surgery: a randomised controlled pilot trial. Int J Surg 20:95-100

25. Terris DJ, Snyder S, Carneiro-Pla D et al (2013) American Thyroid Association statement on outpatient thyroidectomy. Thyroid 23:1193-1202

26. Harding J, Sebag F, Sierra M et al (2006) Thyroid surgery: postoperative hematoma-prevention and treatment. Langenbecks Arch Surg 391:169-173

27. Ozdemir M, Makay O, Icoz G et al (2017) What adds Valsalva maneuver to hemostasis after Trendelenburg's positioning during thyroid surgery? Gland Surg 6:433-436

28. Doran H, England J, Palazzo F (2012) Questionable safety of thyroid surgery with same day discharge. Ann R Coll Surg Engl 94:543-547

Publisher's Note Springer Nature remains neutral with regard to jurisdictional claims in published maps and institutional affiliations. 